\title{
Conducting Polymer- Hydrogel Blends for Electrochemically Controlled Drug Release Devices
}

\author{
Rosângela C. Barthus, Luiz M. Lira and Susana I. Córdoba de Torresi* \\ Instituto de Química, Universidade de São Paulo, CP 26077, \\ 05513-970, São Paulo - SP, Brazil
}

\begin{abstract}
Blendas formadas pela polimerização eletroquímica de pirrol em matriz de acrilamida (hidrogel) foram utilizadas, neste trabalho, como dispositivos para a liberação controlada de drogas. A influência de vários parâmetros nesta síntese, como tipo de matriz de hidrogel e condições de polimerização, foi estudada empregando-se planejamento fatorial fracionário. O objetivo final consistiu na obtenção de um material adequado para liberação baseada no controle de potencial. Para os testes de liberação foi utilizada Safranina, como substância modelo. As curvas de liberação demonstram a potencialidade do uso de tais dispositivos. Estes dispositivos foram também caracterizados por espectroscopia Raman e voltametria cíclica.
\end{abstract}

Blends formed by electrochemical polymerization of polypyrrole (PPy) into polyacrylamide (PAAm) hydrogels were used as devices for controlled drug release. The influence of several parameters in the synthesis, such as type of hydrogel matrix and polymerization conditions was studied by using a fractional factorial design. The final goal was to obtain an adequate device for use in controlled release tests, based on electrochemical potential control. For controlled release tests, Safranin was used as model drug and release curves (amount of drug vs. time) have shown that these blends are promising materials for this use. The optimized blends obtained were characterized by cyclic voltammetry and Raman spectroscopy.

Keywords: factorial design, polypyrrole-polyacrilamide blends, drug delivery, electrochemically controlled release

\section{Introduction}

A new class of materials based on blends formed by conducting polymer-hydrogels has been subject of investigations in several types of researches. ${ }^{1-6}$ This type of material has been classified as a semi-interpenetrating network and displays one of the most exciting synergism in polymer science: the combination of the metallic behavior of conducting polymers ${ }^{7-12}$ with the swelling properties assigned to hydrogels. ${ }^{13}$

In a recent publication the use of a blend formed by polyaniline and polyacrylamide, prepared by electrochemical synthesis, and used as a device for controlled release of drugs was described. ${ }^{2}$ As a continuation of this pioneer study, the synthesis and characterization of another device formed by polypyrrole - polyacrylamide for the same purpose, is shown here. An important fact that is worth to mention is that the use of polypyrrole instead of polyaniline in a blend, opens the

*e-mail: storresi@iq.usp.br possibility of using this material in neutral $\mathrm{pHs}$, overcoming problems related to the fact of polyaniline electroactivity being restricted to acid solutions. The synthesis of an electroactive blend for this application is not trivial and depends on several experimental conditions. The influence of some parameters, i.e., the pore size of hydrogel matrices and ideal polymerization conditions (monomer and electrolyte concentrations, amount of polymerization charge and applied potential), were studied by using a $2^{5-1}$ fractional factorial design. ${ }^{14}$ This type of design is very useful to initially verify the influence of these factors in blends synthesis by means a triage. The ultimate goal was to obtain an electrochemical device able to modulate the drug release rates by switching the potential between positive or negative values extending the well known capabilities of hydrogels for drug release devices. ${ }^{15,16}$

The controlled release tests were carried out by using Safranin (Scheme 1) as model drug. This choice is based on several important characteristics of this compound such as high solubility in water, intense absorption in the visible range and high molar mass. ${ }^{2}$ 
<smiles>Cc1cc2nc3cc(C)c(N)cc3[n+](-c3ccccc3)c2cc1N</smiles>

Scheme 1. Safranin.

The optimized blends suitable to be applied in controlled release tests were characterized by cyclic voltammetry and Raman microscopy.

\section{Experimental}

\section{Reagents and materials}

Pyrrole was purchased form Aldrich (98\% purity) and distilled before use. The following chemicals were used as received: Acrylamide (AAm) (VETEC) N,N'methylenebisacrylamide (MBA) (Invitrogen), potassium peroxodisulfate (Merck), N,N,N',N'-tetramethylethylenediamine (TEMED) (Invitrogen) and potassium chloride (Synth). Purified water from Elga System UHQ was used for all synthesis protocols.

\section{Hydrogel synthesis}

Both, polyacrylamide hydrogels and the electrochemical synthesis of polypyrrole inside the hydrogel matrices, were prepared as described by Wallace et al. ${ }^{6}$ Two different types of hydrogel matrices (pore size) were prepared as follows: different amounts of both acrylamide (AAM) and N,N'methylene-bis-acrylamide (MBA) were added to $1 \mathrm{~mL}$ of purified water $(0.1021 \mathrm{~g}$ of AAm and $0.0056 \mathrm{~g}$ of MBA, for the first hydrogel matrix; $0.0523 \mathrm{~g}$ of AAm and $0.0026 \mathrm{~g}$ of MBA, for the second hydrogel matrix). After mixing, the solution was degassed with nitrogen for $10 \mathrm{~min}$ and then, $10 \mu \mathrm{L}$ of TEMED and $0.0010 \mathrm{~g}$ of potassium peroxodisulfate, were added under stirring. Before gelification, the pre-gel solution was injected in a plastic tube. After the hydrogel formation the material was cut into cylindrical pieces of $50 \mu \mathrm{L}$ and placed in purified water for extracting impurities. The hydrogels formed were used as substrates for the electrochemical synthesis of the conducting polymer.

\section{Synthesis of hydrogel-conducting polymer blends}

For the electrochemical synthesis a platinum wire (0.5 mm diameter) was inserted in the hydrogel matrices that were used as three dimensional working electrodes in a conventional one-compartment electrochemical cell.
A platinum sheet was used as counter electrode and all potentials are referred to $\mathrm{Ag} / \mathrm{AgCl} / \mathrm{Cl}^{-}$(sat) . The electrolytic solution was sodium nitrate where pyrrole was added to achieve the concentration required. Electrochemical polymerizations were performed in an Autolab-PGSTAT30 (ECO CHEMIE) potentiostat/galvanostat and nitrogen was used for degassing the solution. The final blends obtained were placed in purified water for $24 \mathrm{~h}$ in order to eliminate all possible soluble residues.

\section{Fractional factorial design}

The synthesis of conducting polymer-hydrogel blends was evaluated by using a $2^{5-1}$ fractional factorial design. Some additional experiments from a $2^{5}$ factorial design were done in order to confirm some results. The factors studied in two levels designed as (+1) and (-1) were type of hydrogel matrix (pore size) and polymerization conditions. Physically different polyacrylamide hydrogels matrices were synthesized by controlling the amount of acrylamide and the crosslinking agent MBA. The pore sizes $(\xi)$ of each hydrogel matrix were determined by applying the Equilibrium Swelling Theory developed by Peppas and Merril as described in previous publications. ${ }^{2}$ The polymerization conditions evaluated were monomer and electrolyte concentration, amount of charge and potential applied during polymerization. The factors and levels used in this factorial design are shown in Table 1 and the description of all experiments is detailed in Table 2. The experiment was considered as successful when the blend formed was able to release Safranin in a modulated manner depending on the potential applied.

Table1. Factors and levels used in factorial design

\begin{tabular}{lcc}
\hline Factors & Level $(-1)$ & Level $(+1)$ \\
\hline Hydrogel matrix pore size / nm & 0.52 & 2.0 \\
Monomer concentration / $\left(\mathrm{mol} \mathrm{L}^{-1}\right)$ & 0.4 & 0.5 \\
Electrolyte concentration / $\left(\mathrm{mol} \mathrm{L}^{-1}\right)$ & 1.0 & 1.2 \\
Potential applied / V & 0.6 & 0.7 \\
Polymerization charge / C & 8.5 & 10.0 \\
\hline
\end{tabular}

Electrochemical characterization of polypyrrolepolyacrilamide blends

Electrochemical characterization of conducting polymer-hydrogel blends was carried out in a free monomer $1 \mathrm{~mol} \mathrm{~L}-1 \mathrm{NaNO}_{3}$ electrolytic solution by sweeping the potential from $-1.0 \mathrm{~V}$ to $+1.1 \mathrm{~V}$ and using an AutolabPGSTAT30 (ECO CHEMIE) potentiostat/galvanostat. The electrochemical cell used was the same described for the electrochemical polymerization experiments. 
Table 2. Experiments carried out by using factorial design

\begin{tabular}{lccccc}
\hline & $\begin{array}{c}\text { Matrix } \\
\text { Hydrogel } \\
\text { type } \\
\text { (pore size/nm) }\end{array}$ & $\begin{array}{c}\text { Polymerization } \\
\text { Potential } \\
(\mathrm{V})\end{array}$ & $\begin{array}{c}\text { Pyrrole } \\
\text { Monomer } \\
\text { Concentration } \\
\left(\mathrm{mol} \mathrm{L}^{-1}\right)\end{array}$ & $\begin{array}{c}\text { Electrolyte } \\
\mathrm{NaNO}_{3} \\
\text { Concentration } \\
\left(\mathrm{mol} \mathrm{L}^{-1}\right)\end{array}$ & $\begin{array}{c}\text { Charge } \\
\text { Quantity } \\
(\mathrm{C})\end{array}$ \\
\hline 1 & -1 & -1 & -1 & -1 & +1 \\
2 & +1 & -1 & -1 & -1 & -1 \\
3 & -1 & +1 & -1 & -1 & -1 \\
4 & +1 & +1 & -1 & -1 & +1 \\
5 & -1 & -1 & +1 & -1 & -1 \\
6 & +1 & -1 & +1 & -1 & +1 \\
7 & -1 & +1 & +1 & -1 & +1 \\
8 & +1 & +1 & +1 & -1 & -1 \\
9 & -1 & -1 & -1 & +1 & -1 \\
10 & +1 & -1 & -1 & +1 & +1 \\
11 & -1 & +1 & -1 & +1 & +1 \\
12 & +1 & +1 & -1 & +1 & -1 \\
13 & -1 & -1 & +1 & +1 & +1 \\
14 & +1 & -1 & +1 & +1 & -1 \\
15 & -1 & +1 & +1 & +1 & -1 \\
16 & +1 & +1 & +1 & +1 & +1 \\
$* 17$ & -1 & -1 & -1 & -1 & -1 \\
$* 18$ & -1 & +1 & -1 & -1 & -1 \\
$* 19$ & +1 & +1 & -1 & -1 & -1 \\
$* 20$ & +1 & -1 & +1 & -1 & -1 \\
$*$ *2 & +1 & & & &
\end{tabular}

\section{Raman microscopy}

Raman spectroscopy experiments in the microscopic mode were performed in a Renishaw Raman Imaging Microscope System 3000 with an Olympus metallurgical microscope coupled to CCD detector. A He-Ne laser $\left(\lambda_{\mathrm{o}}=623.8 \mathrm{~nm}\right.$, Spectra physics Mod 127) was used as the exciting radiation. Laser power was kept in $0.3 \mathrm{~mW}$ at the sample and the accumulation time was $10 \mathrm{~s}$ for all experiments.

\section{Controlled release tests}

Polypyrrole-polyacrylamide (PPy/PAAm) blends were placed in a $0.01 \mathrm{~mol} \mathrm{~L}^{-1}$ Safranin aqueous solution for $24 \mathrm{~h}$. After this, the blends were taken out of this solution, rinsed with water and transferred to an electrochemical cell containing $20 \mathrm{~mL}$ of $0.1 \mathrm{~mol} \mathrm{~L}^{-1} \mathrm{KCl}$ electrolytic solution. The amount of Safranin released in the neutral solution was followed by UV-Vis spectroscopy using a HP 8453 diode array spectrophotometer. Electro-liberation curves (moles of Safranin $v s$. time) were recorded and the influence of the applied potential $(-0.5 \mathrm{~V}$ and $+0.5 \mathrm{~V})$ on the liberation rate was analyzed.

\section{Results and Discussion}

\section{Fractional factorial design}

The properties of the blends strongly depend on synthesis conditions. By using a $2^{5-1}$ fractional factorial design the influence of empirical parameters like hydrogel porosity and polymerization conditions (amount of charge, applied potential, electrolyte and monomer concentrations) onto the characteristics of the resulting blends were analyzed. The final purpose was to obtain a blend presenting characteristics for being employed as a device for electrochemical controlled release. This characteristic was evaluated by determining the electrochemical control efficiency, defined as $\varphi=\Delta v / v_{\text {red }}$ where $\Delta v=v_{\text {ox }}-v_{\text {red }}$ being $\mathrm{v}_{\text {red }}$ the liberation rate in the re ${ }^{\mathrm{s}}$ lced state and $\mathrm{v}_{\mathrm{ox}}$ the liberation rate in the oxidized state.

From the initial experiments it was observed that the electrochemical polymerization of pyrrole into polyacrylamide presents several particularities. In this study it was verified that only the combination of the factors related to electrochemical polymerization in their lower levels leads to successful synthesis. In the case of charge parameter, it was verified that 8.5 C corresponds to the minimum amount needed to observe electrochemical activity. This is a compromising fact, because increasing the amount of polypyrrole inside the matrix, a loss of the mechanical proprieties of the blends is observed. By using the other experimental parameters, synthetic problems were verified mainly related to not reproducible properties of the blends formed due to the growth of polypyrrole outside the hydrogel.

As a result of using fractional factorial design, some information can be lost because the effect produced, for example, the change in the amount of charge is mixed with the effects produced by the change of the others parameters. To confirm this, some additional experiments in addition of a $2^{5}$ factorial design were carried out. From all, only two experiments (\#2 and \#17) lead to acceptable synthesis conditions. The two blends obtained were denoted as A, in which the hydrogel pore size was $0.52 \mathrm{~nm}$, and $\mathrm{B}$, in which the hydrogel pore size was $2.0 \mathrm{~nm}$. The ideal electrochemical polymerization parameters were $0.4 \mathrm{~mol} \mathrm{~L}^{-1}$ pyrrole, $1.0 \mathrm{~mol} \mathrm{~L}^{-1} \mathrm{NaNO}_{3}, \mathrm{E}_{\text {app }}=+0.6 \mathrm{~V}$ and a final charge of $8.5 \mathrm{C}$.

From the synthesis, it was noted that the growth of the conducting polymer inside the hydrogel matrix was initiated at the platinum electrode and gradually spread out through the hydrogel structure. The distribution of these conducting polymers through the hydrogel depends on the type of hydrogel used, as can be corroborated by Raman spectroscopy, and it will be explained later; here it is important to mention that the polymerization of pyrrole inside the entire hydrogel network will determine the electrochemical control efficiency of the blends. For controlled release tests it was used Safranin as model drug. At first, safranin was incorporated by dipping the hydrogel/ polymer conducting blends in an aqueous safranin solution 
and, due to the interaction based mainly in hydrogen bonds, this substance remains inside the blend.

Figures 1 and 2 show the safranin release profiles for these two devices. It can be seen that, in both cases, in open circuit conditions (OCP) (curves a), the release is mainly governed by diffusion process. When a potential programme is applied (curves b), it is possible to observe that maintaining the potential at $-0,5 \mathrm{~V}$, the liberation rate is always lower than in OCP conditions and it is practically stopped after $c a$. 170 min (blend A) or 210 min (blend B). At this time, the potential is switched to $+0.5 \mathrm{~V}$ initiating the increase of the release rate. Once the released was stopped, potential was changed again to $-0.5 \mathrm{~V}$ and later to $+0.5 \mathrm{~V}$. When a negative potential is applied, no changes in the release rate is observed and by switching to $+0.5 \mathrm{~V}$ the release rate starts increasing again. As observed, both curves are similar but it can be inferred that the applied potential is efficient for controlling the release of safranin because the rate of liberation is modified by changing the potential from

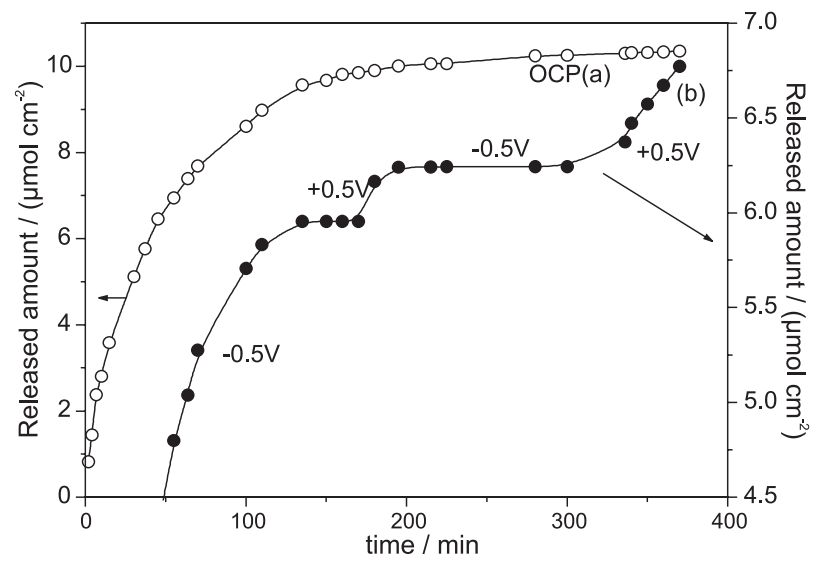

Figure 1. Safranin release curves in $0.1 \mathrm{~mol} \mathrm{~L}-1 \mathrm{KCL}$ from blend A (PPy/PAAm- hydrogel pore size $0.52 \mathrm{~nm}$ ) .

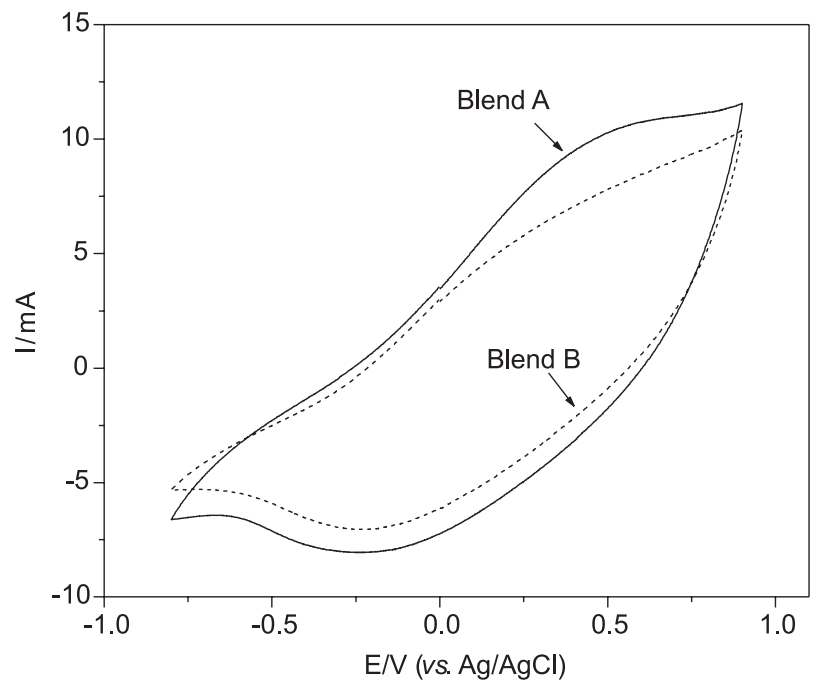

Figure 2. Safranin releases curves (amount released vs. time) in $0.1 \mathrm{~mol} \mathrm{~L}^{-1} \mathrm{KCl}$ from blend B (PPy/PAAm-hydrogel pore size $2.0 \mathrm{~nm}$ ). negative to positive values. On the other hand, it can be clearly noticed the ability of switching off/switching on of the blends. Another point that must be taken into account is the fact that blend A was able to incorporate/release higher amount of safranin than blend B.

To verify an applicability of this type of material, the electrochemical efficiency control ( $Æ)$ defined as $\varphi=\Delta v / v_{\text {red }}$ where $\Delta \mathrm{v}=\mathrm{v}_{\text {ox }}-\mathrm{v}_{\text {red }}$ being $\mathrm{v}_{\text {red }}$ the liberation rate in the reduced state and $v_{\text {ox }}$ the liberation rate in the oxidized state was calculated. It was observed that while the electrochemical efficiency calculated for blend A was 6.4 for the first exchange potential and 24.0 for the second exchange potential, for blend $\mathrm{B}$ it was verified that for the first exchange was 2.5 and for the second was 17.2.

The next section will explain these pointed differences and will provide a molecular interpretation for this set of results based on data obtained by using cyclic voltammetry and Raman spectroscopy.

Characterization of polypyrrole-polyacrylamide blends by cyclic voltammetry

Figure 3 shows the i/E potentiodynamic profiles obtained with polypyrrole-polyacrylamide blend A (hydrogel pore size $0.52 \mathrm{~nm}$ ) and blend $\mathrm{B}$ (hydrogel pore size $2.0 \mathrm{~nm}$ ) in a monomer free $1.0 \mathrm{~mol} \mathrm{~L}^{-1} \mathrm{NaNO}_{3}$ electrolytic solution. It is possible to verify that the cyclic voltammograms show the characteristic features observed for thin polypyrrole films. The anodic and cathodic peaks at $c a .0 .40$ and $-0.25 \mathrm{~V}$ are associated with oxidation/reduction processes which are accompanied by uptake/release of anions which are exchanged in and out of the blend. ${ }^{6}$

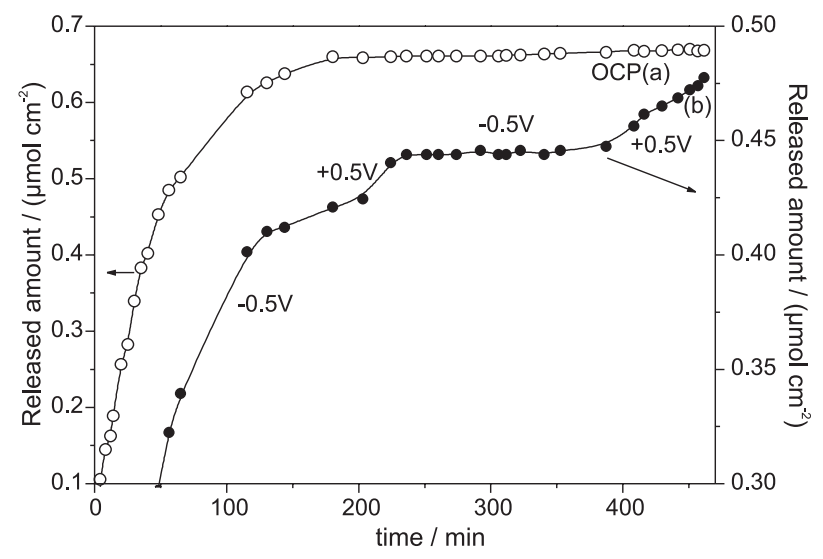

Figure 3. Cyclic voltammograms of blend A (PAAm/Ppy-hydrogel pore size $0.52 \mathrm{~nm}$ ) and blend B (PAAm/PPy-hydrogel pore size $2.0 \mathrm{~nm}$ ) in $1.0 \mathrm{~mol} \mathrm{~L}^{-1} \mathrm{NaNO}_{3}$ electrolytic solution. Scan rate: $0.05 \mathrm{~V} \mathrm{~s}^{-1}$.

It was verified that in spite of the high amount of charge passed $(8.5 \mathrm{C})$ to form the polymer, the electroactivity is 
still very low. The oxidation or reduction charge of the blends measured from the voltammograms and, obviously responsible for electroactivity, corresponds to only $5 \%$ of the total of charge introduced in the blends This must be due to the molecular interactions between the hydrogel matrix and the conducting polymer which are not very strong and do not allow the electrochemical activity of the blend to be more effective, resulting in materials with not so good actuations. This is completely different in the case of polyaniline/polyacrylamide blends, as was reported in a previous publication, ${ }^{2}$ indicating that the electroactivity of conducting polymer/hydrogel blends strongly depends on the chemical nature of the conducting phase and also, it is evident that the distribution of the conducting polymer within the hydrogel matrix is a crucial point that will define the electrochemical properties of the blends.

\section{Characterization of polypyrrole-polyacrylamide blends by using Raman Microscopy}

Raman Microscopy is a very efficient tool that was used in order to elucidate the distribution of the conducting polymer within the hydrogel network. The main advantage of this technique is related to spatial resolution that allows obtaining important information about different domains in the same sample. Figures 4 and 5 show the Raman spectra obtained at three different points of the two blends studied: one near the electric contact (1) and two other points (2-3) at the external border (insert in Figure 4). The comparison of Raman intensities can be performed due to the fact that all spectra were obtained by using the same laser power, accumulation time and number of accumulations.

It is possible to observe that due to the Raman Resonant effect, all spectra show only the characteristic bands of

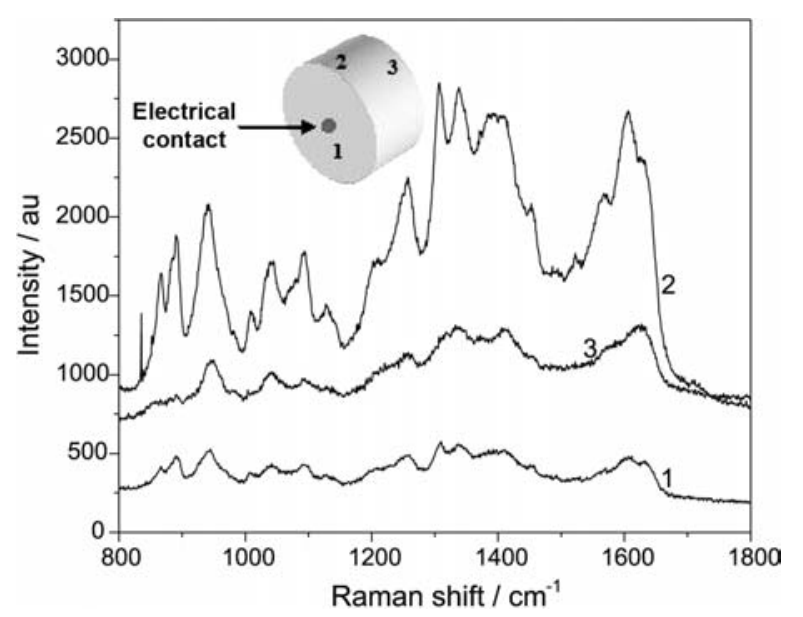

Figure 4. Raman spectra obtained at different points of a blend A formed by polypyrrole inside polyacrilamide matrix ( pore size $0.52 \mathrm{~nm}$ ).

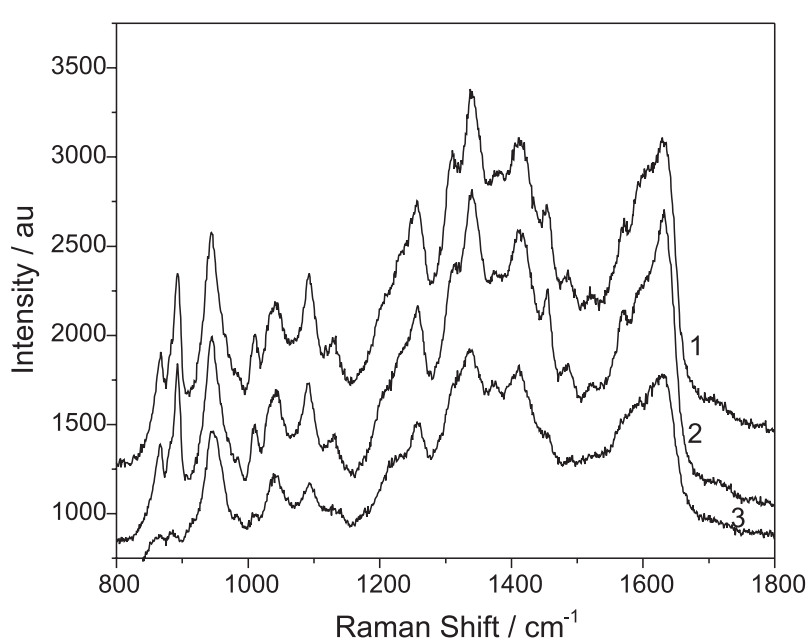

Figure 5. Raman spectra obtained at different points of a blend $B$ formed by polypyrrole inside polyacrilamide matrix (pore size $2.0 \mathrm{~nm}$ ).

polypyrrole, confirming the fact that the electropolymerization inside the hydrogel was successful. By analyzing each spectra and comparing with the others taken at different points of the same sample, it is possible to verify that, a more heterogeneous distribution is observed in blend $\mathrm{A}$ (hydrogel pore size $0.52 \mathrm{~nm}$ ). On the contrary, in blend B (hydrogel pore size $2.0 \mathrm{~nm}$ ) the distribution seems to be more homogeneous. These differences are believed to be due to the pore size that will influence the form in which the conducting polymer grows inside the hydrogel matrix. In case of a blend B, with larger pore size and more flexibility, the distribution of the conducting polymer occurs occupying homogeneously all the hydrogel matrix. The conducting phase is well distributed within the whole hydrogel. For blend A the distribution seems to be concentrated in some regions leading to different conductivity zones in the same material (concentrate in some regions) and due to this fact, results in a more efficient device for controlled release of drugs.

\section{Molecular models for the different blends}

In order to explain the observed differences in the release profiles and put together the electrochemical and spectroscopic information, Figures 6 and 7 present two models for the polypyrrole distribuition in the hydrogel matrix. In Figure 6, a cross section view of the blend formed by the electropolimerization of polypyrrole inside the hydrogel with pore size $0.52 \mathrm{~nm}$ is shown. In this case, Raman microscopy results indicated a more heterogeneous distribution of polypyrrole chains inside the hydrogel matrix with a higher content of PPy in the external border and small amounts in the center. This type of material leads to a more effective safranin electro-release control due to the concentrate pyrrole and them more potential effect. 


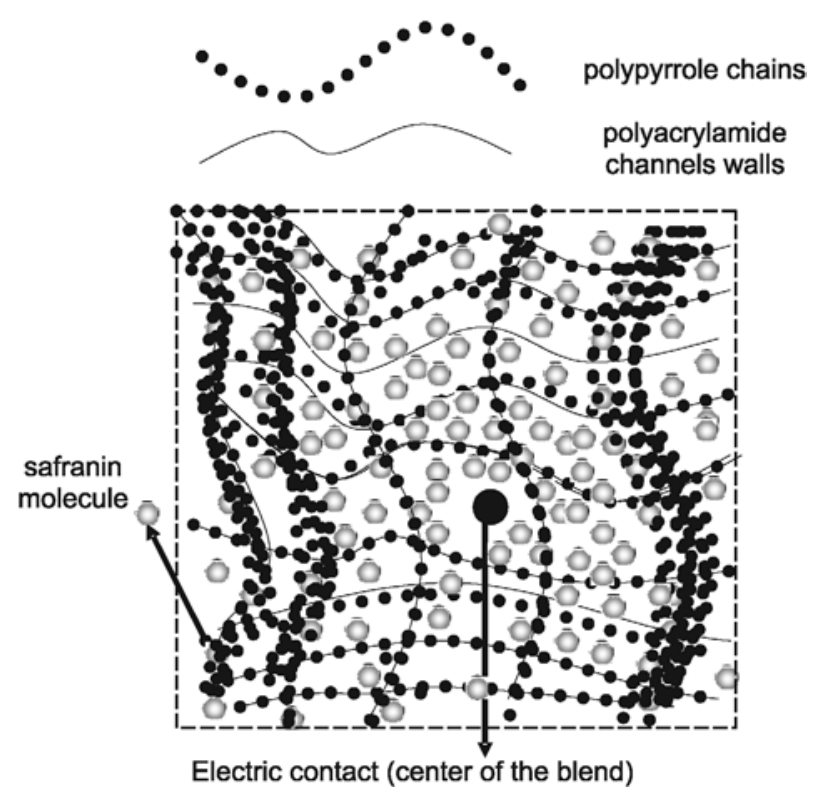

Figure 6. Model for the polypyrrole distribution inside the polyacrylamide network (Matrix A). The hydrogel's porous size is about $0.52 \mathrm{~nm}$.

Figure 7 presents a scheme for the blend formed by polypyrrole and the hydrogel with pore size $2.0 \mathrm{~nm}$. In this case the hydrogel's pore size allows the formation of a material where the conducting polymer can both spread within the structure and accumulate in the free spaces. The distribution of conducting polymer is more effective for the whole hydrogel, but a less pronounced effect in controlled release tests was observed.
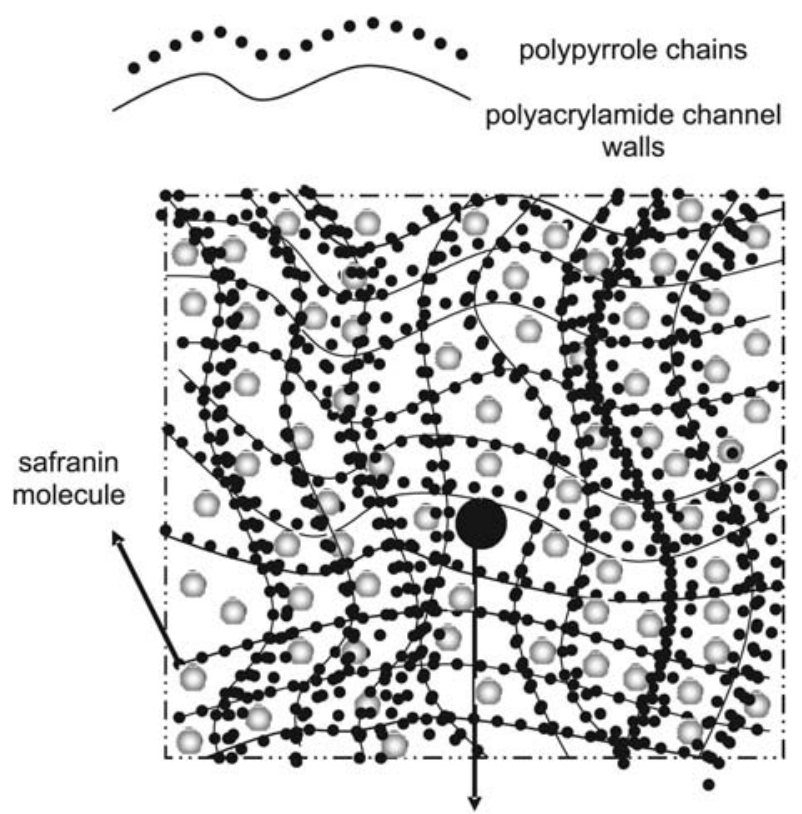

Electric contact (center of the blend)

Figure 7. Model for the polypyrrole distribution inside the polyacrylamide network (Matrix B). The hydrogel's porous size is about $2.0 \mathrm{~nm}$.
It was pointed out that the blend A prepared with the hydrogel-pore size $0.52 \mathrm{~nm}$ absorbs a higher amount of safranin during the swelling process. This can also be related to the heterogeneous polypyrrole distribution because, as many hydrogel's channels are not completely filled with the conducting polymer, the remaining free space (in each channel) can be loaded with safranin molecules. On the other hand, due to a more homogeneous distribution of the conducting phase in the blend B with the hydrogel-pore size $2.0 \mathrm{~nm}$, it is expected that the free volume available to accommodate safranin molecules to be smaller, resulting in a lower safranin amount that can be loaded and then released.

Furthermore, as experimental protocols in the controlled release experiments for the two blends were the same, the differences in the electrochemical control efficiency can be attributed also to the polypyrrole distribuition. At this point it is necessary to discuss the mechanism for the electrochemical release control. It is very well-known that conducting polymers can contract or expand when an electric field is applied. This characteristic is due to the injection of positive charges in the polymeric chains when the material is oxidized and the electrostatic repulsion will produce the expansion of the network. It was verified in an earlier work ${ }^{2}$ for polyaniline/polyacrylamide blends that the electrochemical controlled release is mainly due to the change of volume of the entire material upon changes in its oxidation states. Furthermore, it was observed that the release of either cationic or anionic molecules is increased by applying a positive potential. The oxidation of the conducting phase of the blends provokes an increase of volume, allowing a higher swelling degree and, consequently, a rise in the liberation rate. In the present case the polypyrrole/polyacrylamide blends work in a similar way, causing the liberation of Safranin.

\section{Conclusions}

This paper presents a new class of blends that put together in one material the swelling properties of the hydrogels and electroactivity of conducting polymers. Blends formed by the electropolymerization of polypyrrole inside a threedimensional reticulated polyacrylamide network, have proved to be suitable materials for electrochemically controlled drug release devices and can act in neutral $\mathrm{pH}$ conditions, which is essential for "in vivo" applications. These blends were used as an electrochemically controlled device for the liberation of volumous molecules as safranin due to the available empty space inside the blend that allows storing the dye molecules. The change in the oxidation state of the material provokes physical and chemical changes in the conducting 
polymer network as expansion/contraction and changes in the total charge. These phenomena will be responsible for the electrochemically controlled release of safranin. The relevance of parameters related to the synthesis of the material, composition of the blend, porosity of the hydrogel in release performance was analyzed. In the future devices for electrochemical modulated response, the understanding of the actuation mechanisms at molecular level is a crucial task to optimize the electro-release process.

\section{Acknowledgments}

This work was supported by FAPESP (Proc. No. 03/10015-3), CNPq and Instituto do Milenio de Materiais Complexos network. R.C.B thanks CNPq (Proc. No.150826/2005-2) for the Scholarship granted. We also thank the Laboratório de Espectroscopia Molecular (IQUSP) for providing Raman facilities.

\section{References}

1. Brahim, S.; Narinesingh, D.; Guiseppi-Elle, A.; Electroanalysis 2002, 14, 627.

2. Lira, L.M.; Córdoba de Torresi, S.I; Electrochem. Commun. 2005, 7, 717.

3. Small, C.J.; Too, C.O.; Wallace, G.G; Polym. Gels Netw. 1997, 5,251

4. Moschou, E.A.; Madou, M.J.; Bachas, L.G.; Daunert, S.; Sens. Actuators, B 2006, 115, 379.
5. Brahim, S.; Guiseppi-Elie, A.; Electroanalysis 2005, 17, 556.

6. Kim, B.C.; Spinks, G.M.; Wallace, G.G.; John, R.; Polymer 2000, 41, 1783.

7. Massoumi, B.; Entezami, A.; Eur. Polym. J. 2001, 37, 1015.

8. Shim, W.S.; Lee, Y.H.; Yeo, In-H.; Lee, J.Y.; Lee D.S.; Synth. Met. 1999, 104, 119.

9. Pyo, M.; Maeder, G.; Kenedy, R.T.; Reynolds, J.R.; J. Electroanal. Chem. 1994, 368, 329.

10. Konturi, K.; Penti, P.; Sundlom, G.; J. Electroanal. Chem. 1998, 453, 231.

11. Kontturi, K.; Murtomaki, L.; Pentti, P.; Sundholm, G.; Synth. Met. 1998, 92, 179.

12. Miller, L.L.; Smith, G.A.; Chang, An-C.; Zhou, Q.X.; J. Controll. Rel. 1997, 6, 293.

13. van der Linden, H.J.; Herber, S.; Olthuis, W.; Bergveld, P.; Analyst 2003, 128, 325.

14. Box, G.E.P.; Hunter, J.S.; Hunter, H.G.; Statistics for Experimenters, John Wiley: New York, 1978.

15. Liu, Z.H.; Jiao, Y.P.; Zhang, Z.Y.; J. Appl. Polym. Sci. 2007, $103,3164$.

16. Li, M.G.; Lu, W.L.; Wang, J.C.; Zhang, X.; Zhang, H.; Wang, X.Q.; Wu, C.S.; Zhang, Q.; J. Nanosci. Nanotechnol. 2006, 6, 2874.

Received: July 27, 2007

Web Release Date: December 6, 2007

FAPESP helped in meeting the publication costs of this article. 